\title{
AVALIAÇÃO DOS RESULTADOS DO TRATAMENTO CIRÚRGICO DA RIGIDEZ PÓS-TRAUMÁTICA DO COTOVELO DE PACIENTES ESQUELETICAMENTE MATUROS
}

\author{
EVALUATION OF THE RESULTS OF SURGICAL TREATMENT OF \\ POSTTRAUMATIC STIFFNESS OF THE ELBOW IN SKELETALLY MATURE PATIENTS
}

Alberto Naoki Miyazaki', Marcelo Fregoneze², Pedro Doneux Santos ${ }^{3}$, Luciana Andrade da Silva ${ }^{3}$, Giovanni Di Giunta ${ }^{4}$, Lúcio Norio Watanabe ${ }^{4}$, Sérgio Luiz Checchia ${ }^{5}$

\section{RESUMO}

Objetivo: Avaliar os resultados do tratamento cirúrgico da rigidez pós-traumática do cotovelo em pacientes esqueleticamente maturos. Métodos: Entre outubro de 2000 e outubro de 2007, 45 cotovelos de 45 pacientes foram submetidos a tratamento cirúrgico pelo Grupo de Cirurgia de Ombro e Cotovelo do Departamento de Ortopedia e Traumatologia da Faculdade de Ciências Médicas da Santa Casa de São Paulo. Dez pacientes foram tratados por via artroscópica e o restante por via aberta. O seguimento mínimo foi de seis meses, com média de 22 meses. A idade variou de 17 a 72 anos, com média de 36 anos e três meses. Houve predomínio do sexo masculino em $60 \%$ dos casos. O membro dominante foi acometido em 56,5\%. A avaliação clínica dos resultados foi feita pelos critérios da American $\mathrm{Me-}$ dical Association (AMA), modificada por Bruce, e os do Mayo Elbow Performance Score (MEPS), além da mensuração do ganho do arco de flexoextensão e do arco final de movimento. Resultados: Pelos critérios de AMA, modificados por Bruce, obtivemos $42,2 \%$ de resultados satisfatórios, contra $77,8 \%$ pelos de MEPS. O arco de flexoextensão médio pós-operatório foi de $106^{\circ}$, e média de ganho de amplitude de $46^{\circ}$. A avaliação das variáveis mostrou que pacientes com flexão inicial maior que $90^{\circ}$ obtiveram arco de flexoextensão final maior e, aqueles com extensão inicial menor ou igual a $60^{\circ}$ ganharam maior arco de movimento. Conclusão: O tratamento cirúrgico da rigidez póstraumática do cotovelo em indivíduos esqueleticamente maturos mostrou-se satisfatório pelo método de avaliação do MEPS, mas insatisfatório pelo da AMA. Observamos que os pacientes

\section{ABSTRACT}

Objective: To evaluate the surgical treatment of posttraumatic stiffness of the elbow in skeletally mature patients. Methods: Between October 2000 and October 2007, 45 elbows of 45 patients underwent surgical treatment by the Shoulder and Elbow Surgery Group, Department of Orthopedics and Traumatology, Faculty of Medical Sciences of the Santa Casa de São Paulo. Ten patients were treated by arthroscopy and the remainder by open surgery. Minimum follow-up was six months, with an average of 22 months. Age ranged from 17 to 72 years, with a mean of 36 years and three months. Male were predominant, accounting for $60 \%$ of cases. The dominant limb was involved in 56.5\% of cases. Clinical evaluation of the results was done by the criteria of the American Medical Association (AMA), modified by Bruce, the Mayo Elbow Performance Score (MEPS), and by measuring the gain of the flexo-extension arc and the final range of movement. Results: According to the AMA criteria, modified by Bruce, we have obtained satisfactory results of $42.2 \%$, in contrast to $77.8 \%$ according to the MEPS. The mean postoperative arc of flexo-extension was $106^{\circ}$, with a $46^{\circ}$ mean range gain. The evaluation of the variables showed that patients with an initial flexion arc greater than $90^{\circ}$ obtained higher end flexo-extension, and those with an initial extension less than or equal to $60^{\circ}$ gained a greater range of motion. Conclusion: Surgical treatment of posttraumatic stiffness of the elbow in skeletally mature individuals was satisfactory for the MEPS, but poorly according to the AMA. We observed that patients with flexion greater than $90^{\circ}$ preoperatively evolved with a higher

1 - Professor Assistente e Chefe do Grupo de Ombro e Cotovelo do Departamento de Ortopedia e Traumatologia da Faculdade Ciências Médicas da Santa Casa de São Paulo.

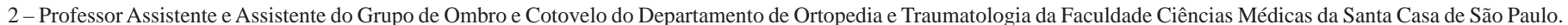

3 - Assistente do Grupo de Ombro e Cotovelo do Departamento de Ortopedia e Traumatologia da Faculdade Ciências Médicas da Santa Casa de São Paulo.

4 - Estagiário do Grupo de Ombro e Cotovelo do Departamento de Ortopedia e Traumatologia da Faculdade Ciências Médicas da Santa Casa de São Paulo.

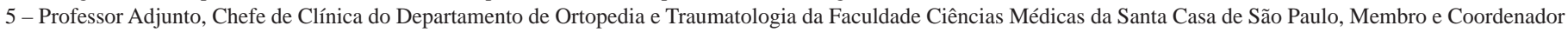
Acadêmico do Grupo de Ombro e Cotovelo.

Correspondência: Rua Dr. Cesário Mota Jr., 112 - Vila Buarque, São Paulo - SP. CEP: 01221-020. E-mail: ombro@ombro.med.br

Trabalho recebido para publicação: 31/08/09 aceito para publicação: 09/03/10. 
com flexão maior que $90^{\circ}$ no período pré-operatório evoluíram com arco de flexoextensão maior após o tratamento cirúrgico, enquanto que os que tinham contratura com extensão menor ou igual a $60^{\circ}$ ganharam maior arco de movimento.

Descritores - Cotovelo; Contratura; Cirurgia; Amplitude de Movimento Articular; Resultado de Tratamento arc of flexo-extension after surgical treatment, while those who had extension contracture of less than or equal to $60^{\circ}$ gained a wider range of motion.

Keyword - Elbow; Contracture; Surgery; Range of Motion, Articular; Treatment Outcome

\section{INTRODUÇÃO}

O cotovelo é uma articulação com grande propensão a evoluir com diminuição da amplitude de movimento (ADM), tanto por causa traumática quanto inflamatória ${ }^{(1)}$. A complexidade desta junta composta por três articulações, a íntima proximidade da cápsula articular com os músculos, a frequência de fraturas cominutas nesta região e ainda a imobilização prolongada utilizada por alguns ortopedistas são fatores que predispõem a rigidez ${ }^{(1-4)}$.

A etiologia da rigidez pós-traumática do cotovelo é multifatorial; a degeneração articular, a ossificação heterotópica, a incongruência articular pós fraturas e a contratura de partes moles periarticulares são achados frequentes ${ }^{(5)}$. De acordo com as condições da musculatura e das estruturas osteoligamentares, pode ser classificada em três tipos: extrínseca, intrínseca e mista ${ }^{(6)}$. A extrínseca é quando a lesão acomete estruturas extraarticulares e é encontrada em pacientes com lesão muscular e/ou da cápsula articular, sequelas de queimaduras, neuropatias compressivas e ossificações heterotópicas. A rigidez intrínseca é causada por alterações que acometem a superfície articular como: consolidação viciosa pós-fraturas, lesões cartilaginosas, aderências intra-articulares, interposição de tecidos periarticulares e formação de osso intra-articular ${ }^{(6-8)}$. A mista é aquela em que há comprometimento tanto do componente intrínseco quanto do extrínseco, e é a mais frequente $\mathrm{e}^{(9)}$.

Morrey et $a l^{(10,11)}$ consideram que a ADM funcional mínima necessária para realizar as atividades da vida diária deva ter um arco entre $130^{\circ}$ de flexão e $-30^{\circ}$ de extensão e $50^{\circ}$ tanto de pronação quanto de supinação, totalizando assim uma amplitude de $100^{\circ}$ tanto no plano sagital como no coronal; indica o tratamento cirúrgico quando a ADM é menor que a funcional.

Outros fatores devem ser considerados no processo de decisão terapêutica, além da ADM, como dor e necessidades individuais de cada paciente. O tratamento não operatório deve ser realizado inicialmente em todos pacientes, por um período mínimo de seis meses, com objetivo de atingir um arco de movimento funcional e indolor; após este período, as medidas fisioterápicas têm resposta inexpressivas, quando deve ser indicada liberação cirúrgica, com exceção das deformidades articulares grosseiras em que a liberação cirúrgica é indicada logo que é feito o diagnóstico ${ }^{(12,13)}$. O tratamento cirúrgico pode ser realizado pelas técnicas artroscópica e aberta, com base no tipo da rigidez e nas características de cada paciente. A artroscopia é geralmente limitada aos casos em que há necessidade apenas de liberação da cápsula articular desde que não haja incongruência articular associada $^{(11,14-17)}$.

O objetivo deste estudo é analisar os resultados obtidos com a liberação cirúrgica, aberta ou artroscópica, nos pacientes esqueleticamente maturos com rigidez pós-traumática do cotovelo.

\section{CASUÍSTICA E MÉTODOS}

Entre outubro de 2000 e outubro de 2007, 45 pacientes foram submetidos ao tratamento cirúrgico da rigidez pós-traumática do cotovelo no Grupo de Cirurgia do Ombro e Cotovelo do Departamento de Ortopedia e Traumatologia da Faculdade de Ciências Médicas da Santa Casa de São Paulo.

Foram incluídos todos os pacientes com limitação da extensão superior a $30^{\circ}$ e/ou flexão inferior a $130^{\circ}$, que não responderam satisfatoriamente ao tratamento fisioterapêutico por um período mínimo de seis meses, conforme proposto por Stans et al ${ }^{(13)}$; exceção feita àqueles que apresentavam consolidação viciosa da fratura ou uma luxação inveterada os quais foram tratados sem respeitar esse período (Tabela 1). Os critérios de exclusão foram outras causas de rigidez, como osteoartrose primária, atrite reumatoide, miosite ossificante, queimaduras etc.

Vinte e sete (60\%) pacientes eram do sexo masculino e 18 (40\%) do feminino. A média de idade na época da cirurgia foi de 36 anos e três meses, variando de 17 a 72 anos. O membro dominante foi acometido em 26 pacientes (56,5\%) (Tabela 1$)$. 
Tabela 1 - Dados epidemiológicos dos pacientes com rigidez pós-traumática do cotovelo.

\begin{tabular}{|c|c|c|c|c|c|c|c|c|c|c|c|c|c|c|}
\hline \multirow[t]{2}{*}{ № } & \multirow[t]{2}{*}{ Iniciais } & \multirow[t]{2}{*}{ Idade } & \multirow[t]{2}{*}{ Sexo } & \multirow[t]{2}{*}{ Dom } & \multirow[t]{2}{*}{ Lesão inicial } & \multicolumn{2}{|c|}{ Conduta inicial } & \multicolumn{3}{|c|}{ Queixa pré-op } & \multicolumn{3}{|c|}{$\begin{array}{c}\text { Rigidez } \\
\text { classificação }\end{array}$} & \multirow{2}{*}{$\begin{array}{c}\text { ADM } \\
\text { Pré-op }\end{array}$} \\
\hline & & & & & & Con & Cir & Dor & Deformidade & Parestesia ulnar & $E$ & 1 & $\mathrm{Mi}$ & \\
\hline 1 & AAJR & 28 & $\mathrm{M}$ & & FAF cotovelo & + & & + & & & & & + & $(110,-60)$ \\
\hline 2 & TMCN & 39 & $\mathrm{~F}$ & & $\mathrm{ft}$ cabeça do rádio & & + & & & & & & + & $(90,-40)$ \\
\hline 3 & LRO & 63 & $\mathrm{~F}$ & & ft-lux inveterada, ft coronóide GII & + & & & & & & & + & $(50,-35)$ \\
\hline 4 & FSP & 72 & $\mathrm{M}$ & + & lux exposta & & + & & + & & & & + & $(90,-90)$ \\
\hline 5 & LFA & 47 & $\mathrm{~F}$ & & ft supra-intercondiliana & & + & & + & & & & + & $(90,-20)$ \\
\hline 6 & FRB & 39 & $\mathrm{M}$ & & ft cabeça de rádio & + & & & & & & & + & $(130,-100)$ \\
\hline 7 & EKS & 29 & $\mathrm{M}$ & + & ft-lux cabeça do rádio & + & & & & & & & + & $(100,-45)$ \\
\hline 8 & VNS & 22 & $\mathrm{~F}$ & & ft supracondiliana & + & & & & & + & & & $(130,-90)$ \\
\hline 9 & DSR & 63 & $\mathrm{~F}$ & + & $\mathrm{ft}$ supracondiliana & & + & & & & + & & & $(120,-40)$ \\
\hline 10 & $\mathrm{LF}$ & 32 & $\mathrm{M}$ & + & ft-lux cabeça do rádio & & + & & & & & & + & $(120,-50)$ \\
\hline 11 & LRP & 43 & $\mathrm{M}$ & + & ft cabeça do rádio & + & & & & & & & + & $(120,-30)$ \\
\hline 12 & RGP & 17 & $\mathrm{M}$ & + & ft supracondiliana exposta & & + & & & & & & + & $(120,-35)$ \\
\hline 13 & EAWG & 54 & $\mathrm{~F}$ & & ft supra-intercondiliana & & + & & + & & & & + & $(100,-80)$ \\
\hline 14 & NEA & 55 & $\mathrm{~F}$ & + & ft côndilo medial & & + & & & & & & + & $(110,-30)$ \\
\hline 15 & JEAP & 35 & $\mathrm{~F}$ & & $\mathrm{ft}$ supracondiliana & & + & & + & & & & + & $(90,-30)$ \\
\hline 16 & RAS & 17 & $\mathrm{M}$ & & $\mathrm{ft}$ supracondiliana & & + & & & & + & & & $(130,-60)$ \\
\hline 17 & WLSA & 30 & $\mathrm{M}$ & & $\mathrm{ft}$ olécrano exposta & & + & & & & & & + & $(100,-45)$ \\
\hline 18 & JSN & 19 & $\mathrm{~F}$ & + & ft cabeça do rádio & + & & & & & & & + & $(110,-50)$ \\
\hline 19 & DGS & 67 & $\mathrm{~F}$ & + & ft tróclea e capítulo & & + & & & & & & + & $(90,-10)$ \\
\hline 20 & JJS & 39 & M & + & $\begin{array}{c}\mathrm{ft} \text { terço proximal antebraço + } \\
\text { lesão neurovascular }\end{array}$ & & + & & & & & & + & $(100,-50)$ \\
\hline 21 & BASS & 41 & $\mathrm{M}$ & + & $\mathrm{ft}$ supracondiliana & + & & & & + & + & & & $(110,0)$ \\
\hline 22 & EGR & 41 & $\mathrm{~F}$ & & ft supracondiliana exposta & & + & & & & & & + & $(90,-80)$ \\
\hline 23 & RCSS & 44 & $\mathrm{M}$ & + & ft supra-intercondiliana exposta & & + & & & & & & + & $(90,-30)$ \\
\hline 24 & TAC & 47 & M & + & $\begin{array}{c}\mathrm{ft} \text { supra-intercondiliana }+\mathrm{ft} \\
\text { ossos antebraço proximal }\end{array}$ & & + & + & & & & & + & $(110,-50)$ \\
\hline 25 & JHP & 48 & $\mathrm{M}$ & & ft supra-intercondiliana & & + & & & & & & + & $(100,-10)$ \\
\hline 26 & DGCS & 34 & $\mathrm{~F}$ & & lux & + & & & & & + & & & $(120,-40)$ \\
\hline 27 & PCA & 32 & $\mathrm{~F}$ & + & $\mathrm{ft}$ cabeça do rádio & + & & & & & & & + & $(100,-40)$ \\
\hline 28 & VMG & 22 & $\mathrm{~F}$ & + & $\operatorname{lux}$ & + & & & & & + & & & $(95,-40)$ \\
\hline 29 & RRRC & 33 & $\mathrm{M}$ & + & ft-lux cabeça do rádio & + & & & & & & & + & $(100,-30)$ \\
\hline 30 & LM & 18 & $\mathrm{M}$ & + & ft-lux epicondilo medial & + & & & & + & + & & & $(130,-40)$ \\
\hline 31 & RFA & 29 & $\mathrm{M}$ & & ft cabeça do rádio & + & & & & & & & + & $(100,-100)$ \\
\hline 32 & LAE & 51 & $\mathrm{M}$ & + & ft-lux cabeça do rádio & & + & & + & & & & + & $(110,-30)$ \\
\hline 33 & GTD & 19 & $\mathrm{M}$ & + & ft olécrano exposta & & + & & & & & & + & $(100,-30)$ \\
\hline 34 & ASO & 29 & M & & $\begin{array}{l}\text { lesão condral, ft avulsão } \\
\text { epicôndilo medial }\end{array}$ & + & & + & & & & + & & $(120,-30)$ \\
\hline 35 & GMP & 37 & $\mathrm{M}$ & + & ft supra-intercondiliana & & + & & & + & & & + & $(120,-30)$ \\
\hline 36 & LLBP & 33 & $\mathrm{~F}$ & & $\mathrm{ft}$ capítulo & & + & & & & & & + & $(90,-90)$ \\
\hline 37 & VCDS & 24 & $\mathrm{M}$ & + & lux & + & & & & & + & & & $(120,-90)$ \\
\hline 38 & JCS & 32 & $\mathrm{M}$ & + & $\mathrm{ft}$ Monteggia & + & & & + & + & & & + & $(110,-20)$ \\
\hline 39 & MMB & 37 & $\mathrm{M}$ & + & lux & + & & & & & + & & & $(120,-80)$ \\
\hline 40 & LCS & 39 & $\mathrm{M}$ & + & ft cabeça do rádio e coronóide & + & & & & & & & + & $(110,-60)$ \\
\hline 41 & PCM & 33 & $\mathrm{~F}$ & + & ft-lux cabeça do rádio & & + & + & & & & & + & $(90,-80)$ \\
\hline 42 & $\mathrm{IM}$ & 23 & $\mathrm{M}$ & + & ft supra-intercondiliana exposta & & + & & & & & & + & $(130,-80)$ \\
\hline 43 & ADCM & 42 & $\mathrm{~F}$ & & ft cabeça do rádio+ulna & & + & & & & & & + & $(130,-65)$ \\
\hline 44 & LSF & 33 & $\mathrm{~F}$ & & $\mathrm{ft}$ condilo lateral & & + & & & & & & + & $(120,-40)$ \\
\hline 45 & EFDJ & 31 & $\mathrm{M}$ & & lux & + & & & & + & & & + & $(120,-30)$ \\
\hline $\mathrm{T}$ & & & & 26 & & 20 & 25 & 4 & 6 & 5 & 9 & 1 & 35 & \\
\hline
\end{tabular}

Legenda: №: número, Dom: dominância, op: operatória, ADM: amplitude de movimento (flexoextensão), Con: conservador, Cir: cirúrgico, E: extrínseca, I: intrínseca, Mi: mista, M: masculino, F: feminino, FAF: ferimento por arma de fogo, ft: fratura, lux: luxação, T: total. 
A avaliação no período pré-operatório consistiu em anamnese detalhada, exame físico geral e ortopédico e radiografias simples nas incidências em anteroposterior (AP) e perfil, além das incidências de perfil em flexão e extensão máximas (Figuras 1A e 1B), visando identificar possíveis pontos de bloqueio ósseo. Outros exames de imagem, como tomografia computadorizada e ressonância magnética (Figuras 1C e 1D), foram realizados de acordo com a necessidade de cada caso.

O tempo decorrido entre a lesão inicial e o tratamento cirúrgico da rigidez variou entre seis meses e 32 anos, com média de dois anos. Vinte e cinco (55,6\%) pacientes foram operados previamente para tratamento da lesão traumática inicial (Tabela 1).

A queixa principal estava relacionada à limitação de movimento, sendo que quatro pacientes $(8,9 \%)$ tinham dor associada, e seis (13,3\%) queixavam-se de deformidade. Em cinco casos (11,1\%) havia sintomas relacionados ao nervo ulnar (Tabela 1).

A ADM tanto de flexoextensão como de pronossupinação de ambos os cotovelos foi medida com goniômetro padrão. O arco de flexoextensão também foi avaliado radiograficamente na incidência de perfil em flexão e extensão máximas no período pré e pós-operatório imediato, ainda sob efeito anestésico (bloqueio de plexo braquial), e nos retornos ambulatoriais (Figuras 1E e 1F).

O diagnóstico do trauma inicial variou, sendo os de maior incidência: 13 casos (28,9\%) de fratura suprain- tercondiliana, seis $(13,3 \%)$ de luxações sem fratura, seis $(13,3 \%)$ fraturas da cabeça do rádio e cinco $(11,1 \%)$ fraturas-luxação da cabeça radial. Quanto à etiologia da rigidez: um caso (2,2\%) foi classificado como intrínseca, nove (20\%) como extrínseca e 35 (77,8\%) como mista (Tabela 1).

Quando da via aberta, o acesso posterior foi utilizada em 26 (57,8\%), lateral em cinco (11,1\%), lateral associada à medial em quatro $(8,9 \%)$ para realizar a neurólise e anteriorização do ulnar, e a via medial foi acesso único em um caso (caso 45), em que havia ossificação somente medial. Em 10 pacientes (22,2\%), o procedimento cirúrgico foi realizado por via artroscópica, associado a uma via medial nos casos em que o nervo ulnar fosse explorado (Tabela 2).

Todos os pacientes foram submetidos à capsulectomia anterior e posterior independentemente do tipo de rigidez e da via de acesso utilizada. Foram associados outros procedimentos de acordo com a necessidade, como: retirada de material de síntese em 14 casos (31,1\%), exérese da cabeça do rádio em nove (20\%), ressecção da ponta do olécrano em oito (17,8\%), ressecção de ossificação em cinco $(11,1 \%)$ e anteriorização do nervo ulnar em 11 (24,4\%). Este foi anteriorizado quando havia queixa de parestesia pré-operatória (cinco casos - 11,1\%) e quando se notava tensão do mesmo após a liberação articular (seis casos - 13,3\%) (Tabela 2).
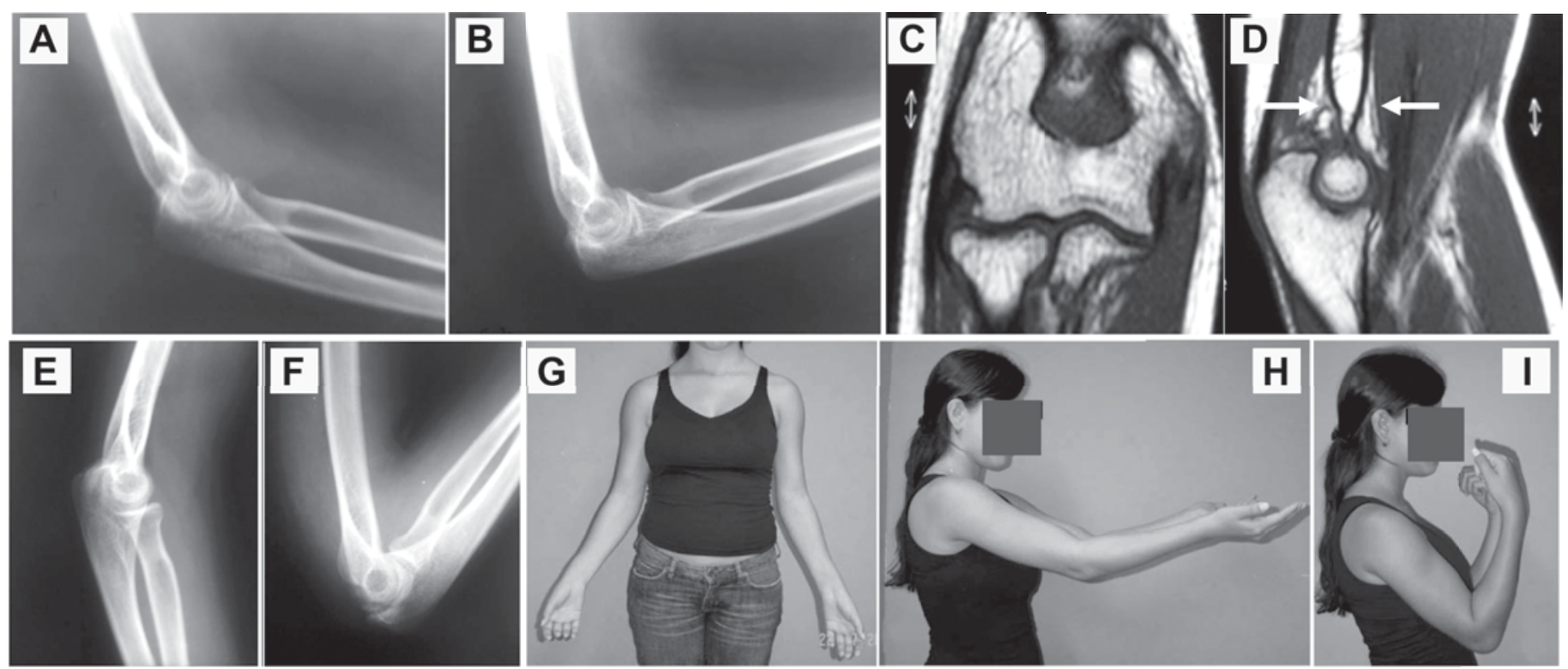

Figura 1 - Imagens referentes ao paciente de número $28 \mathrm{com}$ resultado satisfatório. A e B - Imagens radiográficas préoperatórias do cotovelo direito, em perfil, em extensão $\left(-40^{\circ}\right)$ e flexão máximas $\left(95^{\circ}\right)$. C e D - Imagem de ressonância magnética mostrando superfície articular em bom estado e espessamento capsular anterior e posterior (setas brancas) do cotovelo direito. E e F - Imagens radiográficas pós-operatórias em perfil em extensão $\left(-30^{\circ}\right)$ e flexão máximas $\left(130^{\circ}\right)$. G, H e I - Imagens de frente e perfil em extensão e flexão máximas na última consulta. 
Tabela 2 - Procedimentos realizados no tratamento cirúrgico da rigidez pós-traumática do cotovelo e resultados.

\begin{tabular}{|c|c|c|c|c|c|c|c|c|c|c|c|c|c|c|c|c|c|c|}
\hline \multirow[t]{2}{*}{ № } & \multirow{2}{*}{$\begin{array}{c}\Delta \mathrm{T} \\
\text { (meses) }\end{array}$} & \multirow{2}{*}{ Acesso } & \multirow{2}{*}{$\begin{array}{l}\text { Ant } \mathrm{n} \\
\text { ulnar }\end{array}$} & \multirow{2}{*}{ RMS } & \multicolumn{3}{|c|}{ Ressecção } & \multirow{2}{*}{$\begin{array}{c}\text { Seg } \\
\text { (meses) }\end{array}$} & \multicolumn{4}{|c|}{ MEPS } & \multicolumn{4}{|c|}{ AMA } & \multirow[t]{2}{*}{ ADM } & \multirow[t]{2}{*}{ Complicações } \\
\hline & & & & & $\begin{array}{l}\text { Cabeça } \\
\text { do rádio }\end{array}$ & Ossificação & $\begin{array}{l}\text { Ponta do } \\
\text { olécrano }\end{array}$ & & Ex & B & $\mathrm{Re}$ & $\mathrm{Ru}$ & Ex & B & $\operatorname{Re}$ & $\mathrm{Ru}$ & & \\
\hline 1 & 9 & $\mathrm{p}$ & + & & & & & 24 & & & + & & & & & + & $(120,-20)$ & $\begin{array}{c}\text { parestesia do } \\
\text { n. ulnar }\end{array}$ \\
\hline 2 & 4 & 1 & & + & & & & 13 & + & & & & & + & & & $(110,-20)$ & \\
\hline 3 & 6 & $\mathrm{p}$ & & & & & & 27 & + & & & & & + & & & $(140,-20)$ & \\
\hline 4 & 4 & $\mathrm{I}+\mathrm{m}$ & & & & & & 74 & & & & + & & & & + & $(80,-50)$ & cotovelo luxado \\
\hline 5 & 6 & $\mathrm{p}$ & & + & & & & 36 & + & & & & + & & & & $(130,0)$ & \\
\hline 6 & 12 & $\mathrm{p}$ & & & + & & + & 12 & + & & & & & & & + & $(130,0)$ & \\
\hline 7 & 12 & $\mathrm{a}$ & & & + & & & 6 & & + & & & & & & + & $(130,-35)$ & \\
\hline 8 & 121 & $p$ & & & & & + & 43 & & & + & & & & & + & $(120,-50)$ & \\
\hline 9 & 7 & $p$ & & + & & & & 40 & + & & & & & + & & & $(140,-10)$ & \\
\hline 10 & 8 & 1 & & & + & & & 36 & & + & & & & & & + & $(135,-25)$ & \\
\hline 11 & 8 & $\mathrm{a}$ & & & + & & & 30 & + & & & & & + & & & $(130,0)$ & \\
\hline 12 & 22 & $\mathrm{p}$ & & & & & + & 58 & & + & & & & & + & & $(120,-10)$ & $\begin{array}{c}\text { neurotmese do } \\
\text { n. radial }\end{array}$ \\
\hline 13 & 5 & $\mathrm{p}$ & & + & & & & 38 & & + & & & & + & & & $(130,-10)$ & \\
\hline 14 & 13 & $\mathrm{p}$ & & & & & + & 36 & + & & & & & & + & & $(110,-20)$ & \\
\hline 15 & 6 & $p$ & + & + & & & & 7 & & + & & & & & + & & $(110,-30)$ & \\
\hline 16 & 54 & $\mathrm{p}$ & & & & & + & 6 & + & & & & & + & & & $(140,-20)$ & \\
\hline 17 & 17 & $p$ & & + & & & + & 32 & + & & & & & + & & & $(130,-15)$ & \\
\hline 18 & 15 & $p$ & & & + & & + & 19 & & & + & & & & & + & $(100,-15)$ & \\
\hline 19 & 12 & $\mathrm{p}$ & & + & & & & 38 & & + & & & & & + & & $(120,0)$ & $\begin{array}{c}\text { parestesia do } \\
\text { n. ulnar }\end{array}$ \\
\hline 20 & 13 & $p$ & & + & & & & 16 & & & + & & & & & + & $(130,-50)$ & \\
\hline 21 & 384 & $a+m$ & + & & & & & 36 & & + & & & & & & + & $(130,0)$ & \\
\hline 22 & 14 & $\mathrm{p}$ & & & + & + & & 11 & & & + & & & & & + & $(100,-40)$ & $\begin{array}{c}\text { parestesia do } \\
\text { n. ulnar }\end{array}$ \\
\hline 23 & 12 & $\mathrm{p}$ & & & & & & 23 & & + & & & & & & + & $(100,-20)$ & $\begin{array}{c}\text { parestesia do } \\
\text { n. ulnar }\end{array}$ \\
\hline 24 & 42 & $p$ & & & & & & 8 & & & + & & & & & + & $(110,-10)$ & \\
\hline 25 & 16 & $p$ & & + & & & & 10 & & & + & & & & & + & $(110,-20)$ & \\
\hline 26 & 6 & a & & & & & & 18 & + & & & & + & & & & $(140,-10)$ & \\
\hline 27 & 8 & a & & & + & & & 18 & & + & & & & & & + & $(120,-30)$ & \\
\hline 28 & 10 & $a+m$ & + & & & & & 17 & + & & & & & + & & & $(130,-30)$ & $\begin{array}{c}\text { neurotmese } \\
\text { do n. radial e } \\
\text { axoniotmese do } \\
\text { n. mediano }\end{array}$ \\
\hline 29 & 4 & a & & & & & & 30 & & + & & & & & + & & $(130,-10)$ & \\
\hline 30 & 19 & a & + & & & & & 24 & + & & & & & + & & & $(140,-30)$ & \\
\hline 31 & 7 & $p$ & + & & & + & & 27 & & & + & & & & & + & $(90,-10)$ & \\
\hline 32 & 11 & $\mathrm{I}+\mathrm{m}$ & & & & & & 24 & + & & & & & + & & & $(130,0)$ & \\
\hline 33 & 6 & $\mathrm{p}$ & & + & & & & 23 & + & & & & + & & & & $(130,-10)$ & \\
\hline 34 & 10 & $\mathrm{a}$ & & & & & & 13 & & + & & & & & + & & $(140,-35)$ & \\
\hline 35 & 14 & $\mathrm{p}$ & + & + & & & & 35 & & & + & & & & + & & $(140,-5)$ & $\begin{array}{c}\text { parestesia do } \\
\text { n. ulnar }\end{array}$ \\
\hline 36 & 10 & $\mathrm{p}$ & + & & & & & 20 & & + & & & & & & + & $(100,-10)$ & \\
\hline 37 & 5 & a & & & & & & 20 & + & & & & + & & & & $(140,0)$ & \\
\hline 38 & 240 & $\mathrm{I}+\mathrm{m}$ & + & & + & & & 14 & + & & & & + & & & & $(140,0)$ & \\
\hline 39 & 14 & 1 & & & & + & & 17 & & + & & & + & & & & $(140,-10)$ & \\
\hline 40 & 7 & 1 & & & & + & & 15 & + & & & & & + & & & $(130,-20)$ & \\
\hline
\end{tabular}




\begin{tabular}{|c|c|c|c|c|c|c|c|c|c|c|c|c|c|c|c|c|c|c|}
\hline \multirow[t]{2}{*}{ № } & \multirow[t]{2}{*}{$\begin{array}{c}\Delta \mathrm{T} \\
\text { (meses) }\end{array}$} & \multirow[t]{2}{*}{ Acesso } & \multirow[t]{2}{*}{$\begin{array}{l}\text { Ant } \mathrm{n} \\
\text { ulnar }\end{array}$} & \multirow[t]{2}{*}{ RMS } & \multicolumn{3}{|c|}{ Ressecção } & \multirow{2}{*}{$\begin{array}{c}\text { Seg } \\
\text { (meses) }\end{array}$} & \multicolumn{4}{|c|}{ MEPS } & \multicolumn{4}{|c|}{ AMA } & \multirow[t]{2}{*}{ ADM } & \multirow[t]{2}{*}{ Complicações } \\
\hline & & & & & $\begin{array}{c}\text { Cabeça } \\
\text { do rádio }\end{array}$ & Ossificação & $\begin{array}{l}\text { Ponta do } \\
\text { olécrano }\end{array}$ & & Ex & B & $\mathrm{Re}$ & $\mathrm{Ru}$ & Ex & B & $\operatorname{Re}$ & $\mathrm{Ru}$ & & \\
\hline 41 & 4 & $\mathrm{I}+\mathrm{m}$ & + & & & & & 18 & & + & & & & & + & & $(130,-10)$ & \\
\hline 42 & 34 & $p$ & & + & & & + & 8 & + & & & & & & + & & $(120,-40)$ & \\
\hline 43 & 7 & $\mathrm{p}$ & & + & + & & & 13 & & + & & & & & & + & $(120,-50)$ & \\
\hline 44 & 18 & I & & + & & & & 12 & + & & & & + & & & & $(140,-10)$ & $\begin{array}{c}\text { parestesia do } n . \\
\text { ulnar }\end{array}$ \\
\hline 45 & 7 & $\mathrm{~m}$ & + & & & + & & 8 & + & & & & + & & & & $(150,0)$ & \\
\hline$T$ & & & 11 & 14 & 9 & 5 & 8 & & 20 & 15 & 9 & 1 & 8 & 11 & 9 & 17 & & \\
\hline
\end{tabular}

Fonte: SAME DOT-ISCMSP.

Legenda: №: número, $\triangle T$ : tempo entre a lesão inicial e o tratamento da rigidez, Ant: anteriorização, n: nervo, RMS: retirada de material de síntese, Seg: segmento, ADM: amplitudende de movimento (flexoextensão), Ex: excelente, B: bom, Re: regular, Ru: ruim, p: posterior, l: lateral, m: medial, a: artroscópica, T: total, MEPS: Mayo Elbow Perfomance Score, AMA: American Medical Association.

A analgesia pós-operatória foi realizada pela administração de analgésicos endovenosos e por via oral. A fisioterapia foi iniciada no primeiro dia pós-operatório com mobilização passiva, sendo que a ativa se iniciou na quarta semana e exercícios com carga somente após a oitava semana.

Os pacientes foram reavaliados segundo protocolos de avaliação funcional propostos por Morrey et al $\mathrm{l}^{(11)}$ (MEPS) e os critérios da AMA modificados por Bruce et $a^{(18)}$ (Tabela 2). A ADM foi registrada em todas as consultas pós-operatórias com a utilização de um goniômetro (Figuras 1G, 1H e 1I) (Tabela 1).

Neste estudo listamos variáveis pré-operatórias que poderiam indicar um melhor ou pior prognóstico para o tratamento da rigidez pós-traumática do cotovelo. Estas variáveis foram: idade, dominância, sexo, tempo entre lesão inicial e tratamento da rigidez, tipo e tratamento do trauma inicial, e subdividimos as contraturas em quatro grupos, segundo o grau de mobilidade articular (Quadro 1).

Quadro 1. Grupos de contratura de acordo com a limitação da ADM.

\begin{tabular}{|c|c|}
\hline Grupo & Grau de mobilidade articular \\
\hline I & flexão $\leq 90^{\circ}$ \\
\hline II & flexão $>90^{\circ}$ \\
\hline III & extensão $\leq-60^{\circ}$ \\
\hline IV & extensão $>-60^{\circ}$ \\
\hline
\end{tabular}

Essas variáveis, então, foram comparadas quanto aos resultados finais obtidos, no intuito de definir algum fator prognóstico para o tratamento cirúrgico da rigidez.

Utilizamos o programa SPSS (Statistical Package for Social Sciences) versão 13.0, aplicando o teste de Mann-Whitney e de Kruskal-Wallis para avaliar se as variáveis interfiriam no resultado, sendo que o primeiro teste foi usado para duas variáveis (dominância, sexo, flexão $\leq 90^{\circ}$ e extensão $\leq-60^{\circ}$ e tratamento inicial) e o segundo para mais de duas (idade, tempo entre a lesão inicial e o tratamento da rigidez e tipo de trauma inicial). Utilizamos o teste dos postos sinalizados de Wilcoxon para avaliar a diferença entre os resultados obtidos pelas escalas de MEPS e AMA, e para verificar diferença entre a flexão, extensão e arco de flexoextensão inicial e final. Em todos os testes foi utilizado valor de $p \leq 0,05$ como estatisticamente significante.

\section{RESULTADOS}

O tempo de seguimento pós-operatório médio foi de 22 meses, variando entre seis e 74 meses.

A medida clínica da média de amplitude do arco de flexoextensão aumentou de $60^{\circ}$, no período pré-operatório, para $106^{\circ}$ na última avaliação. Houve diferença estatisticamente significante $(\mathrm{p}<0,001)$ quanto a melhora da flexão, da extensão e do arco de movimento.

De acordo com o protocolo de avaliação funcional proposto pela AMA modificado por Bruce, foram verificados oito casos classificados como excelentes (17,8\%), 11 bons (24,4\%), nove regulares (20\%) e 17 ruins (37,8\%), com média de 80,62 pontos. Segundo o MEPS, verificamos 20 casos classificados como excelentes (44,5\%), 15 casos bons (33,3\%), nove regulares (20\%) e um ruim (2,2\%), com média de 85,11 pontos. As avaliações pelos critérios de AMA e MEPS foram comparadas e verificou-se que o primeiro é muito mais rigoroso, tanto na distribuição de pontos, com média de 80,62 contra 85,11 do MEPS, quanto no número de resultados satisfatórios $42,2 \%$ contra $77,8 \%$, com diferença estatisticamente significante $(\mathrm{p}<0,001)$.

Cinco pacientes evoluíram com parestesia no território ulnar. Houve dois casos em que ocorreu lesão neurológica durante a cirurgia, um em uma operação aberta 
(nervo radial) e outro em uma artroscopia (nervo radial e mediano), sendo ambas diagnosticadas e tratadas após o procedimento.

A maioria das variáveis analisadas no período préoperatório não mostrou diferença estatisticamente significante que indicasse como fator prognóstico, com exceção dos grupos II e III (Tabelas 3 e 4).

Tabela 3 - Análise estatística comparando resultado do tratamento da rigidez do cotovelo entre os grupos I e II.

\begin{tabular}{l|c|c|c}
\hline & \multicolumn{2}{|c|}{ Média } & p-valor \\
\hline & Grupo I: $\leq 90^{\circ}$ & Grupo II: $>90^{\circ}$ & \\
\hline Arco F-E & $86^{\circ}$ & $111^{\circ}$ & 0,048 \\
\hline Ganho F-E & $56^{\circ}$ & $44^{\circ}$ & 0,408 \\
\hline MEPS & $78^{\circ}$ & $87^{\circ}$ & 0,24 \\
\hline AMA & $74^{\circ}$ & $82^{\circ}$ & 0,281 \\
\hline
\end{tabular}

Fonte: SAME DOT-ISCMSP.

Legenda - F-E: flexoextensão, MEPS: Mayo Elbow Performance Score, AMA: American Medical Association.

Tabela 4 - Análise estatística comparando resultado do tratamento da rigidez do cotovelo entre os grupos III e IV.

\begin{tabular}{l|c|c|c}
\hline & \multicolumn{2}{|c|}{ Média } & p-valor \\
\hline & $\begin{array}{c}\text { Grupo III: } \leq \\
-60^{\circ}\end{array}$ & $\begin{array}{c}\text { Grupo IV: } \\
-60^{\circ}\end{array}$ & \\
\hline Arco F-E & $95^{\circ}$ & $112^{\circ}$ & 0,126 \\
\hline Ganho F-E & $64^{\circ}$ & $37^{\circ}$ & 0,011 \\
\hline MEPS & $79^{\circ}$ & $88^{\circ}$ & 0,107 \\
\hline AMA & $75^{\circ}$ & $83^{\circ}$ & 0,185 \\
\hline
\end{tabular}

Fonte: SAME DOT-ISCMSP.

Legenda - F-E: flexoextensão, MEPS: Mayo Elbow Performance Score, AMA: American Medical Association.

\section{DISCUSSÃO}

O tratamento cirúrgico da rigidez do cotovelo em adultos é bem estabelecido, com bons resultados na maioria dos pacientes. Relata-se que $90 \%$ dos pacientes apresentam ganho de ADM e mais de 50\% retornam a um arco funcional de movimento ${ }^{(4,6,12,19)}$. Em nossa série, 53,3\% dos pacientes atingiram o arco funcional e apenas quatro pacientes $(8,9 \%)$ obtiveram um ganho inferior a $10^{\circ}$ de flexoextensão, portanto observamos resultados semelhantes à literatura.

Conforme os trabalhos publicados, em relação à causa da rigidez, encontramos uma predominância do tipo mista $(84,7 \%)$ em detrimento da intrínseca e extrínseca $^{(1,20)}$. Ainda similar à literatura encontramos predominância de fratura supraintercondiliana de úmero e da cabeça do rádio como fatores etiológicos de rigidez pós-traumática ${ }^{(3,4,6,21-23)}$.

Quanto ao tratamento, assim como na literatura, utilizamos predominantemente as vias de acesso posterior
(57,8\%) e lateral (20,0\%). Nossa opção pela via artroscópica $(22,2 \%)$ ocorreu nos casos com menor comprometimento articular em que estava indicada apenas a liberação capsular ${ }^{(4,7,12,22,24,25)}$.

No presente estudo verificamos um ganho médio de flexoextensão de $46^{\circ}$, estando dentro do ganho médio descrito na literatura que varia de $40^{\circ}$ a $59^{\text {o(7,12,20-24) }}$.

Turchin et al ${ }^{(26)}$ compararam cinco sistemas de avaliação funcional, incluindo o MEPS, e relataram grande discrepância entre todos os métodos, sugerindo que a disparidade decorre da avaliação de diferentes quesitos por cada método e da atribuição de valores proporcionalmente distintos ao mesmo item de avaliação. Consideramos o método de avaliação da AMA o que melhor retrata o resultado do tratamento da rigidez, por dar maior importância para a ADM, enquanto o de MEPS valoriza principalmente o fator relacionado à dor.

Um caso teve resultado ruim pelos dois métodos, o de número 4, cujo diagnóstico inicial era de luxação exposta inveterada associada a uma infecção latente. Foi realizada uma redução cruenta associada à liberação capsular e retensionamento ligamentar lateral e, durante o intraoperatório, já era observada uma instabilidade com o cotovelo em $30^{\circ}$ de extensão e supinação. Durante o acompanhamento pós-operatório evoluiu com recidiva da infecção e perda da redução, sendo então indicada a artrodese do cotovelo, porém o paciente recusou-se a ser submetido a tal procedimento (Figura 2).

As lesões neurovasculares são descritas na literatura como complicações do tratamento cirúrgico da rigidez de cotovelo. Morrey ${ }^{(12)}$ relata que os três principais nervos do cotovelo podem sofrer injúria durante o ato operatório ou no processo de reabilitação, referindo o nervo ulnar como sendo o mais acometido, podendo ocorrer em $10 \%$ dos casos operados. Cohen e Hastings ${ }^{(22)}$ relataram que a complicação mais comum é a parestesia transitória no território do ulnar, justificada pela manipulação cirúrgica, edema e fibrose no túnel cubital e pelo ganho da flexão causando tensão no nervo, encontrando em seu estudo a incidência de 13,6\%. A neuropraxia do ulnar foi encontrada em cinco pacientes $(11,1 \%)$ em nossa série após o tratamento operatório da rigidez, três casos tiveram melhora sem nova abordagem, e dois casos persistiram com sintomatologia mesmo após a anteriorização em uma nova operação.

A literatura relata que o nervo radial e seu ramo interósseo posterior estão sujeitos a lesões, seja por 

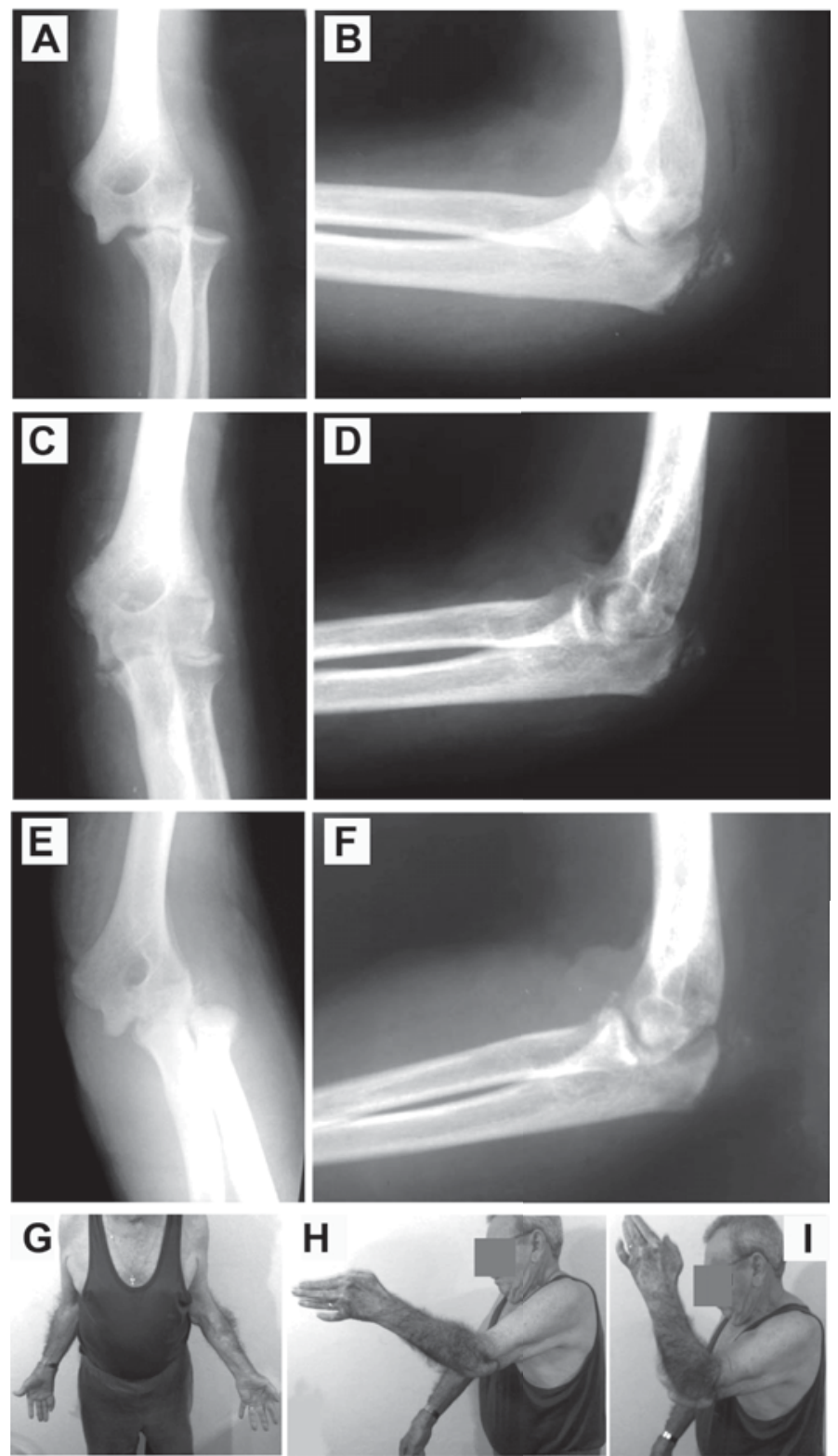

Figura 2 - Imagens referentes ao paciente de número 4, que teve um mau resultado. A e B - Imagem radiográfica em AP e perfil pré-operatória mostrando luxação lateral do cotovelo esquerdo. C e D - Imagem radiográfica pós-operatória, em AP e perfil, do cotovelo esquerdo mostrando redução da luxação. E e $\mathrm{F}$ - Imagem radiográfica do cotovelo esquerdo, em AP e perfil, com recidiva da luxação. G, H e I - Imagens de frente e perfil com flexão e extensão máxima do cotovelo esquerdo do paciente ao final do seguimento.

excessiva compressão pelos afastadores no acesso lateral ou durante a confecção do portal anterolateral na cirurgia artroscópica $^{(1,27)}$. Haapaniemi et al ${ }^{(28)}$ ainda relataram um caso em que houve transecção dos nervos radial e mediano durante liberação artroscópica para tratamento da rigidez. Tivemos dois pacientes com lesões neurológicas: casos 12 e 30. No caso 12 ocorreu neurotmese do radial durante cirurgia aberta, sendo realizado enxerto com nervo sural após três meses; no caso 30 houve axoniotmese do mediano e neurotmese do radial durante artroscopia, sendo então submetido à neurólise do nervo mediano e enxertia com nervo sural no radial após um mês.

Realizamos um estudo estatístico em que tentamos identificar fatores prognósticos para um maior ganho de ADM. Encontramos apenas que os pacientes do grupo III apresentavam maior ganho de ADM, e que pacientes do grupo II apresentaram um arco de flexoextensão final maior, pela possibilidade de uma maior gravidade da rigidez. Estes dados não puderam ser comparados com a literatura, pois não há este tipo de estudo até a presente data.

Os demais fatores como idade, sexo, dominância, tempo entre lesão e tratamento, conduta de tratamento do trauma inicial, tipo de lesão e a presença de contratura em flexão ou extensão não estabeleceram alteração do prognóstico da liberação da rigidez de cotovelo. Ring et $a l^{(24)}$ também não encontraram relação entre idade, sexo, mecanismo de trauma e tratamento inicial como fatores que alterassem os resultados pelas escalas de DASH, MEPS e ASES.

\section{CONCLUSÃO}

Verificamos que o tratamento cirúrgico da rigidez do cotovelo, em pacientes esqueleticamente maduros promoveu uma melhora da ADM.

Pacientes com flexão maior que $90^{\circ}$ obtiveram arco de flexoextensão final maior.

Pacientes que tinham contratura em extensão menor ou igual a $60^{\circ}$ obtiveram ganho maior de movimento.

Observamos que os critérios de avaliação utilizados apresentam diferenças, sendo que o critério de AMA modificado por Bruce se mostrou mais rigoroso que o de MEPS.

\section{REFERÊNCIAS}

1. Morrey BF. Posttraumatic stiff elbow. Clin Orthop Relat Res. 2005;431:26-34.

2. Modabber MR, Jupiter JB. Reconstruction for post-traumatic conditions of the elbow joint. J Bone Joint Surg Am. 1995;77(9):1431-46.

3. Motta GR, Motta LAJ, Mendes HMM. O acesso lateral para tratamento da contratura pós-traumática do cotovelo. Rev Bras Ortop. 1995;30(9):627-32.

4. Urbaniak JR, Hansen PE, Beissinger SF, Aitken MS. Correction of post-traumatic flexion contracture of the elbow by anterior capsulotomy. J Bone Joint Surg Am. 1985;67(8):1160-4.

5. Lindenhovius ALC, Jupiter JB. The posttraumatic stiff elbow: a review of the literature. J Hand Surg. 2007;32(10):1605-23. 
6. Morrey BF. Post-traumatic contracture of the elbow. Operative treatment, including distraction arthroplasty. J Bone Joint Surg Am. 1990;72(4):601-18.

7. Park MJ, Kim HG, Lee JY. Surgical treatment of post-traumatic stiffness of the elbow. J Bone Joint Surg Br. 2004;86(8):1158-62.

8. Bruno RJ, Lee ML, Strauch RJ, Rosenwasser MP. Posttraumatic elbow stiffness: evaluation and management. J Am Acad Orthop Surg. 2002;10(2):106-16.

9. Jupiter JB. Assessment and management of the stiff elbow. J Musculosket Med. 2005;22(2):692-8

10. Morrey BF, Askew RPT, An KN, Chao EY. A biomechanical study of functional elbow motion. J Bone Joint Surg Am. 1981;63(6):872-7.

11. Morrey BF, An KN, Chao, EYS. Functional evaluation of the elbow. In: Morrey BF. The elbow and its disorders. Philadelphia: W.B. Saunders; 1993. p. 86-97.

12. Morrey BF. Surgical treatment of extraarticular elbow contracture. Clin Orthop Relat Res. 2000;370:57-64.

13. Stans AA, Maritz NGJ, O'Driscoll SW, Morrey BF. Operative treatment of elbow contracture in pacients twenty-one years of age or younger. J Bone Joint Surg Am. 2002;84(3):382-7.

14. Wada T, Ishii S, Usui M, Miyano S. The medial approach for operative release of post-traumatic contracture of the elbow. J Bone Joint Surg Br. 2000;82(1):68-73.

15. Barron OA, Catalano LW. The stiff elbow: diagnosis and treatment. Curr Opin Orthop. 2002;13(4):323-7.

16. Field LD, Savoie FH 3rd. The arthroscopic evaluation and management of the elbow trauma and instability. Op Tech Sports Med. 1998;5(1):22-8.

17. Jones GS, Savoie FH 3rd. Arthroscopic treatment of flexion contractures of the elbow. Arthroscopy. 1993;9(3):277-83.
18. Bruce HE, Harvey JPJ, Wilson J. Monteggia fractures. J Bone Joint Surg Am. 1974;56(8):1563-76.

19. Husband JB, Hasting $H$. The lateral approach for operative release of posttraumatic contracture of the elbow. J Bone Joint Surg Am. 1990;72(9):1353-8.

20. Cikes A, Jolles BM, Farron A. Open elbow arthrolysis for posttraumatic elbow stiffness. Orthop Trauma. 2006:20(6):405-9.

21. Sharma S, Rymaszewski LA. Open arthrolysis for post-traumatic stiffness of the elbow. J Bone Joint Surg Br. 2007;89(6):778-81.

22. Cohen MS, Hastings H. Post-traumatic contracture of the elbow: operative release using a lateral collateral ligament sparing approach. J Bone Joint Surg Br. 1998;80(5):805-12.

23. Tan V, Daluiski A, Simic P, Hotchkiss RN. Outcome of open release for posttraumatic elbow stiffness. J Trauma. 2006;61(3);673-8.

24. Ring D, Adey L, Zurakowski D, Jupiter JB. Elbow capsulectomy for posttraumatic elbow stiffness. J Hand Surg Am. 2006;31(8):1264-71.

25. Mansat $P$, Morrey BF. The column procedure: a limited lateral approach for extrinsic contracture of the elbow. J Bone Joint Surg Am. 1998;80(11):1603-15.

26. Turchin DC, Beaton DE, Richards RR. Validity of observer-based aggregate scoring systems as decriptors of elbow pain function, and disability. J Bone Jonit Surg Am. 1998;80(2):154-62.

27. O'Driscoll SW, Morrey BF. Artroscopy of the elbow: diagnostic and therapeutic benefits and hazards. J Bone Joint Surg Am. 1992;74(1):84-94.

28. Haapaniemi T, Berggren M, Adolfsson L. Case report: Complete transaction of the medial and radial nerves during arthroscopic release of post-traumatic elbow contracture. Arthroscopy.1999;15(7):784-7. 